\title{
CONCENTRAÇÃO DE MICRONUTRIENTES EM MÃES E SEUS RECÉM-NASCIDOS POR OCASIÃO DO PARTO
}

\section{MOTHER'S AND NEWBORN'S PLASMATIC CONCENTRATION OF MICRONUTRIENTS AT THE MOMENT OF CHILDBIRTH}

\author{
Ciro João Bertoli ${ }^{1,2}$ \\ Claudio Leone ${ }^{3}$ \\ Virginia B.V. Junqueira ${ }^{4}$ \\ Francisco Roque Carrazza (in memoriam) ${ }^{1}$
}

Bertoli, CJ et al. Concentração de micronutrientes em mães e seus recém-nascidos por ocasião do parto. Rev. Bras. Cresc. e Desenv. Hum. 2010; 20(2): 270-281.

\section{Resumo:}

Trata-se de estudo transversal com uma amostra sequencial de conveniência de 73 gestantes, não fumantes, sem intercorrências durante a gravidez e seus recém-nascidos normais, objetivando comparar concentrações plasmáticas de retinol, $\beta$ caroteno, licopeno, $\alpha$ tocoferol e cobre entre mães e recém-nascidos. Colheu-se sangue da parturiente e do cordão umbilical (após dequitação) para dosagem dos micronutrientes. Retinol, $\beta$ caroteno, licopeno e $\alpha$ tocoferol foram dosados por HPLC e o cobre por espectrofotometria de absorção atômica. Para análise foram separados 2 grupos:- grupo 1, 38 gestantes com suplementação vitaminico-mineral pelo menos no último trimestre da gravidez e grupo 2, 35 gestantes que não haviam recebido suplementação. As comparações estatísticas foram pelo teste $t$ de Student ou pelo teste de Wilcoxon, com um $\alpha$ crítico de 0,05 . Os dois grupos de mães eram símiles quanto a idade, paridade, IMC e a baixa ingestão de calorias e de micronutrientes. Os grupos de recém-nascidos não evidenciaram diferença quanto a sexo, peso, comprimento e perímetro cefálico. As concentrações plasmáticas maternas do grupo 1 foram maiores para o retinol $(\mathrm{p}=0,0034)$ e a tocoferol $(\mathrm{p}=0,0279) . \beta$ caroteno, licopeno e cobre não evidenciaram diferenças entre os dois grupos de mães. Nos dois grupos de recém-nascidos as concentrações de retinol, tocoferol e cobre foram semelhantes e praticamente não houve concentrações detectáveis de licopeno e $\alpha$ caroteno. Em ambos os grupos retinol, a tocoferol e cobre apresentaram maiores concentrações nas mães do que nos recém-nascidos, $\mathrm{p}=$ $0,0003, \mathrm{p}=0,0001, \mathrm{p}=0,0001$, respectivamente. Conclui-se que as concentrações nos recém-nascidos foram sempre menores do que as maternas, independentemente da ingestão e da suplementação durante a gravidez.

Palavras-chave: gestantes; recém-nascidos; sangue de cordão; micronutrientes; cobre; licopeno; $\beta$ caroteno; $\alpha$ tocoferol; retinol.

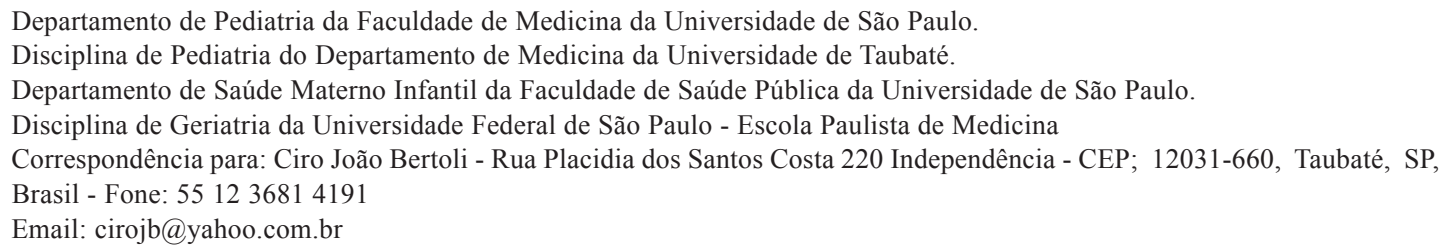




\begin{abstract}
:
Cross sectional study of a sequential convenience sample of 73 non smokers' pregnant women with uncomplicated pregnancies and their normal newborns. The aim of the study was to compare plasmatic concentrations of retinol, $\beta$-carotene, licopen and $\alpha$-tocopherol between mothers and their infants. Blood was obtained from parturient and umbilical cord for micronutrient analysis. HPLC was used for retinol, $\alpha$-carotene, licopen and $\alpha$-tocopherol analysis. Atomic absorption spectrophotometry was used for copper measurements. The sample was divided into 2 groups: group 1, with 38 women that had received vitamin and mineral supplementation during the last gestational trimester and group 2, with 35 women who have had no supplementation. Statistic comparison used Student $t$ test or Wilcoxon test (alpha $=0.05)$. The groups of mothers were similar regarding age, parity, BMI and ingestion (low) of calories and micronutrients. The newborn groups had no differences according to sex, head circumference, weight and length. Plasmatic concentrations in group 1 of mothers were greater for retinol $(p=0.0034)$ and $\alpha$-tocopherol $(p=0.0279)$. $\beta$-carotene, licopen and cupper showed no differences between the groups at mothers. In both newborn groups concentration of retinol, $\alpha$-tocopherol and copper were quite similar and there was no clearly detectable licopen and ${ }^{2}$ carotene. In both, retinol, $\alpha$-tocopherol and cupper showed larger concentration in mothers than in newborns (respectively $\mathrm{p}=0.0003$ and $\mathrm{p}=0.0001$ ). The conclusion is that newborn concentrations were systematically smaller than their mothers', independently of ingestion or supple-mentation during pregnancy.
\end{abstract}

Key words: retinol (vitamin A); $\beta$-caroten; licopen; $\alpha$-tocopherol (vitamin E); cupper; newborns; pregnant women.

\section{INTRODUÇÃO}

Micronutrientes, como as vitaminas e os oligoelementos, ou elementos traço, são substâncias requeridas pelo organismo em pequenas quantidades para o seu funcionamento normal. Dentre estes estão as vitaminas lipossolúveis, como o retinol (vit $\mathrm{A}$ ), $\beta$ caroteno, licopeno, vitamina $\mathrm{D}, \alpha$ tocoferol (vit E) e os oligoelementos como: zinco, cobre, selênio, manganês, crômio, molibdênio e ferro ${ }^{1,2}$. Os grupos populacionais de risco elevado para desenvolver deficiências destes micronutrientes são as crianças, por seu crescimento rápido, e as gestantes ${ }^{2,3}$. A gravidez representa o período nutricional mais sensível de todo o ciclo da vida, sua adequação pode resultar em maior benefício na diminuição da mortalidade materna, fetal e infantil, além de beneficiar a longo prazo, a saúde da população como um todo. Existe correlação entre a dieta da gestante e as condições clínicas do recém-nascido ${ }^{4,5}$.

O retinol é fundamental para muitas funções, incluindo visão, reprodução, crescimento, proliferação e diferenciação celular ${ }^{6}$. Excesso ou deficiência do retinol e dos carotenóides afeta adversamente a embriogênese $\mathrm{e}^{7-9}$. Anormalidades obstétricas, malformações fetais, restrição do crescimento intra-uterino (RCIU) e aborto espontâneo foram mais observados em animais submetidos à hipo ou à hipervitaminose $\mathrm{A}^{9,10}$. Baixas concentrações de retinol no recémnascido estão associadas à broncodisplasia pulmonar, aumento da morbimortalidade por doenças infecciosas e anormalidades de células $\mathrm{T}$ em idades maiores ${ }^{7,11}$.

Seu excesso pode ter efeitos teratogênicos, sugerindo existir interrelação do metabolismo da vitamina A entre a mãe, a placenta e o feto. O retinol e seu metabolismo têm 
sido pouco estudados em mulheres nutridas e com gravidez normal, embora haja grande número de investigações em condições patológicas $^{7}$. Os estudos iniciais com os carotenóides $\beta$ caroteno e licopeno - consideravam que eram importantes como percursores do retinol, mas estudos mais recentes apontam para efeitos protetores mesmo sem se transformarem em retinol. Sua função é a de agir como antioxidantes e/ou quelantes do oxigênio li$v \mathrm{re}^{12-14}$. A deficiência de retinol é considerada problema de saúde pública nos países em desenvolvimento ${ }^{6,10,15}$.

A vitamina $E$ tem seu papel bem definido, protegendo os lipides das membranas celulares do dano oxidativo. É também importante na regulação do metabolismo do ácido nucléico e na síntese de enzimas. No recémnascido, a deficiência de vitamina $\mathrm{E}$ tem sido associada à hemorragia intracraniana, fibroplasia retrolental, hiperbilirrubinemia, trombocitose e anemia hemolítica ${ }^{16-18}$.

O cobre é considerado um elemento traço essencial envolvido em múltiplos sistemas enzimáticos, incluindo a resposta imune. O feto recebe o cobre através da placenta desde o início da gestação, mas maiores quantidades são transferidas durante o último trimestre de gestação ${ }^{19,20}$.

Embora os mecanismos de passagem transplacentária dos micronutrientes não estejam totalmente elucidados, várias teorias são propostas, tais como a de difusão passiva, a favor de gradiente de concentração, ou de transporte acoplados a outras substâncias. As vitaminas lipossolúveis e o cobre sofrem processos de passagem transplacentária semelhantes, dependentes do gradiente, com concentrações menores nos recém-nascidos ${ }^{21-23}$.

Considerando as possíveis conseqüências das deficiências de micronutrientes em gestantes com aporte limítrofe, mesmo que não apresentem manisfestações clínicas, é relevante estudar os níveis séricos destes micronutrientes: retinol, $\beta$-caroteno, lico- peno, $\alpha$ tocoferol e cobre, no binômio mãe/ recém-nascido.

Desta maneira, o objetivo é de verificar as relações existentes entre estas concentrações de micronutrientes em mães e recém-nascidos no momento do parto.

\section{MÉTODO}

Trata-se de um estudo de corte transversal, com coleta de sangue de mães e do cordão umbilical no momento do parto, durante o ano de 1999, em uma amostra seqüencial de conveniência, na cidade de Taubaté, Estado de São Paulo.

Para o cálculo da amostra considerouse que o teste deveria ser capaz de dectetar uma diferença de 0,5 desvio padrão entre as médias comparadas pelo teste $t$ de Student, para dados independentes considerando um $\alpha$ de $5 \%$ e poder de teste de $90 \%$.

O tamanho da amostra estimado como necessário foi igual a 31 pares de mães e recém nascidos em cada grupo.

Partindo da possibilidades de perdas inerentes ao processo de coleta, transporte e a quantidade de soro para a realização de todas as dosagens, decidiu-se adicionar $30 \%$ a mais em cada grupo, de forma que, o tamanho da amostra a ser inicialmente captada em cada grupo foi fixado em 40 pares de mães e recém nascidos, para ao final se obter no mínimo 31 pares de mães e recém nascidos em cada grupo.

A amostra final foi de 73 parturientes, dentre as 94 possíveis com gestação a termo e sem intercorrências no decorrer da mesma. As parturientes foram classificadas em dois grupos: grupo 1 (G1), 38 mães que haviam recebido regularmente suplemento vitamínico-mineral no último trimestre da gravidez. Grupo 2( G2), 35 gestantes, sem seguimento pré-natal e que não haviam recebido suplemento vitamino-mineral em nenhum período da gestação. O suplemento vitamino-mineral era pa- 
dronizado e continha: retinol 5000 UI, Ácido fólico $1 \mathrm{mg}$, Acetato de tocoferol $30 \mathrm{UI}$, Ácido ascórbico $100 \mathrm{mg}$, Cianocobalamina $12 \mathrm{mcg}$, zinco $25 \mathrm{mg}$ e cobre $2 \mathrm{mg}$.

Do total dos casos coletados foram excluídas 21 gestantes ( $22,3 \%$ ), em decorrência de problemas com a quantidade, a conservação e/ou o transporte do material coletado, por causa da distância dos laboratórios em que as análises foram realisadas.

O G1 era composto por 38 gestantes e apresentava uma média de idade de 24,1 anos e desvio padrão(dp) de 4,0 anos. O índice de massa corpórea (IMC) médio do grupo era de $24,9( \pm 5,5)$.

O G2, 35 gestantes, tinha uma média de idade de 22,9 anos $( \pm 3,9)$. O IMC médio deste grupo era de $24,3( \pm 5,0)$. Não havia diferença em relação à paridade entre os dois grupos.

O G1, apresentou maior prevalência de partos cesareos ( tabela 1 ).

Os dois grupos de recém-nascidos eram semelhantes quanto à distribuição por sexo e medidas de comprimento, perímetro cefálico e peso ao nascer (Tabela2).

As amostras de sangue das mães foram coletadas após admissão no estudo, através do venocat utilizado para infusão de soro fisiológico na gestante em trabalho de parto. Foi utilizada seringa descartável de $10 \mathrm{ml}$ para retirar parte da mistura soro-sangue e, em seguida, uma seringa de $20 \mathrm{ml}$ de êmbolo plástico e com $2 \mathrm{ml}$ de heparina sódica para colher o sangue. Esta seringa era imediatamente recoberta com papel escuro e acondicionada em caixa de isopor, para evitar a exposição à luz. Após a dequitação da placenta coletava-se sangue do cordão, com os mesmos cuidados.

O material coletado era levado ao laboratório do Hemocentro do Hospital Universitário de Taubaté, que dispunha de equipamentos adequados para sua centrifugação. Neste, as amostras eram transferidas para tubos siliconizados de 10 $\mathrm{ml}$ e centrifugadas imediatamente a 3000 rotações por minuto, durante 10 minutos. O plasma era separado em dois lotes de tubos de Eppendorff, adequadamente identificados, envoltos em papel de alumínio e mantidos a $-80^{\circ} \mathrm{C}$. Os lotes foram transportados em caixas de isopor com gelo seco para o Laboratório de Vitaminas da UNIFESP - Escola Paulista de Medicina para as dosagens de retinol, $\beta$ caroteno, licopeno, $\alpha$ tocoferol e para o Laboratório do Instituto da Criança Prof. Pedro de Alcantara do Hospital das Clinicas da Faculdade de Medicina da Universidade de São Paulo, para a dosagem do cobre.

\section{DOSAGENS}

As dosagens foram realizadas por lotes. O retinol, $\beta$ caroteno, licopeno e $\alpha$ tocoferol, foram determinados após extração por Cromatografia Líquida de Alta Eficiência (HPLC), em equipamento modelo Waters515. O cobre foi determinado por espectrofotometria de absorção atômica, em equipamento Gemini AA 12 - 1475(Soft Varian). Foram aceitos os valores plasmáticos com coeficiente de variação de $5 \%$, para HPLC e Espectrofotometria de Absorção Atômica. Todas as dosagens foram realizadas em duplicata.

\section{RECORDATÓRIO ALIMENTAR}

A ingestão alimentar das parturientes foi avaliada por meio do recordatório de 24 horas realizado por nutricionista treinada e a sua análise foi feita com o programa de computador de Apoio à Nutrição, da UNIFESP [PROGRAMA DE APOIO À NUTRIÇÃO - Centro de Informática em Saúde (CIS) Escola Paulista de Medicina. Universidade Federal de São Paulo - versão 2.5]. 


\section{ANÁLISE ESTATÍSTICA}

As variáveis classificatórias foram apresentadas em tabelas de contingência, contendo freqüências absolutas (n) e relativas (\%), e a comparação das proporções nos grupos com e sem suplementação foi realizada pelo teste do $\chi^{2}$ ou o Exato de Fisher.

As variáveis contínuas foram apresentadas em tabelas, contendo médias e desvios padrão ou medianas com valores mínimos e máximos. A comparação das médias dos grupos com e sem suplementação foi realizada pelo teste $\mathrm{t}$ de Student. As variáveis que não apresentaram distribuição normal foram comparadas pelo teste da soma de postos de Wilcoxon. O nível de significância adotado $(\alpha)$ foi de $5 \%$.
O estudo foi aprovado pela CAPPESQ, Comissão para Analíse Ética de Projetos de Pesquisa do Hospital das Clínicas da FMUSP e Comissões de Ética e Pesquisa dos demais hospitais. Todas as pacientes autorizaram individualmente suas participações no estudo, após tomar conhecimento das informações contidas no termo de consentimento livre e esclarecido.

\section{RESULTADOS}

Como pode ser observado na tabela 1 , não havia diferença entre os dois grupos de mães quanto à paridade, entretanto observouse maior proporção de partos cesáreos no grupo suplementado.

Tabela 1: Antecedentes de paridade e tipo de parto, segundo os grupos do estudo.

\begin{tabular}{|c|c|c|c|}
\hline & G1 & G2 & $\mathbf{p}$ \\
\hline \multicolumn{4}{|l|}{ Paridade } \\
\hline 1 & $25(65,8 \%)$ & $16(45,7 \%)$ & \\
\hline 2 & $7(18,4 \%)$ & $9(25,7 \%)$ & $0,211^{*}$ \\
\hline 3 & $6(15,8 \%)$ & $10(28,6 \%)$ & \\
\hline \multicolumn{4}{|l|}{ Tipo de parto } \\
\hline cesário & $28(73,7 \%)$ & $15(42,9 \%)$ & $0,007 * *$ \\
\hline normal & $10(26,3 \%)$ & $20(57,1 \%)$ & \\
\hline
\end{tabular}

Quanto às características dos recém nascidos, a tabela 2 evidencia que não havia diferença estatisticamente significante entre os dois grupos de estudo.

No que se refere à ingestão média diária de calorias, cobre e das vitaminas retinol e $\alpha$ tocoferol não se observou diferenças estatisticamente significantes entre os dois grupos de gestantes (tabela 3).
Quanto às concentrações plasmáticas médias de retinol observou-se que o G1, grupo de mães que recebeu suplementação, apresentou valores mais elevados do que o G2 e inclusive do que as concentrações dos seus recémnascidos (tabela 4).

A tabela 5 mostra que não houve diferenças nas concentrações plasmáticas médias de licopeno entre os dois grupos de mães. Ape- 
nas um dos recém-nascidos apresentou nível serico detectável de licopeno.

A tabela 6 mostra concentrações plasmáticas médias de $\beta$ caroteno apenas em sete mães, em ambos os grupos, as úni- cas que apresentaram níveis detectáveis. Os níveis médios obeservados foram semelhantes. Não foi detectado $\beta$ caroteno no sangue de cordão de nenhum dos recémnascidos.

Tabela 2: Caracterização dos recém-nascidos, segundo os grupos de estudo.

\begin{tabular}{llll}
\hline & G1 & G2 & p \\
\hline Sexo & & & \\
Feminino & $17(44,7 \%)$ & $19(54,3 \%)$ & $0,485^{*}$ \\
\multicolumn{1}{c}{ Masculino } & $21(55,3 \%)$ & $16(45,7 \%)$ & \\
\hline Comp $(\mathrm{cm})^{\star}$ & $49,2 \pm 1,86$ & $48,2 \pm 2,16$ & $0,068^{* *}$ \\
PC $(\mathrm{cm})^{\star}$ & $34,9 \pm 1,41$ & $34,7 \pm 1,68$ & $0,592^{* *}$ \\
Peso $(\mathrm{kg})^{\star}$ & $3,29 \pm 0,40$ & $3,51 \pm 0,49$ & $0,061^{* *}$ \\
\hline média $\pm \mathrm{dp}$ & Comp $=$ comprimento & PC = perímetro cefálico &
\end{tabular}

* teste Exato de Fischer $\quad * *$ teste $\mathrm{t}$ de Student para amostras não independentes

Tabela 3: Ingestão materna diária de calorias, retinol, átocoferol e cobre segundo os grupos de estudo.

\begin{tabular}{lccc}
\hline & $\begin{array}{c}\text { G1 } \\
(\mathbf{n}=38)\end{array}$ & $\begin{array}{c}\text { G2 } \\
(\mathbf{n}=35)\end{array}$ & $\mathbf{p}$ \\
\hline quilocalorias* & $1604 \pm 768$ & $1842 \pm 859$ & $0,227^{\star}$ \\
retinol( $\mu \mathrm{g})^{*}$ & $989 \pm 700$ & $1704 \pm 1607$ & $0,247^{\star}$ \\
$\alpha$ tocoferol(mg)** & $7(1-18)$ & $9(1-31)$ & $0,307^{\bullet}$ \\
cobre(mg)** & $1,3(0,0-5,6)$ & $0,4(0,1-1,4)$ & $0,297^{\bullet}$ \\
\hline
\end{tabular}

* Média \pm desvio padrão ** Mediana (mínimo - máximo)

- teste $\mathrm{t}$ de Student para amostras não independentes "• teste de Wilcoxon

Tabela 4: Concentrações plasmáticas médias ( $\mathrm{mmol}$ / L) de retinol, segundo os grupos de estudo.

\begin{tabular}{lccc}
\hline & $\begin{array}{c}\text { G1 } \\
(\mathbf{n}=\mathbf{3 6})\end{array}$ & $\begin{array}{c}\mathbf{G} \mathbf{2} \\
(\mathbf{n}=\mathbf{3 2})\end{array}$ & $\mathbf{p}$ \\
\hline Mãe & $0,65 \pm 0,52^{*}$ & $0,35 \pm 0,30^{*}$ & $0,0034^{\bullet \bullet}$ \\
$\mathrm{RN}$ & $0,45 \pm 0,31^{*}$ & $0,31 \pm 0,24^{*}$ & $0,0752^{\bullet \bullet}$ \\
$\mathrm{P}$ & $0,0003 \mathrm{~s}$ & $0,4624 \mathrm{~s}$ & \\
\hline
\end{tabular}

* Média \pm desvio padrão ${ }^{\bullet}$ teste $t$ de Student para amostras não independentes

- teste $\mathrm{t}$ de Student para amostras independentes 
Tabela 5: Concentrações plasmáticas ( $\mathrm{mmol} / \mathrm{L}$ ) de licopeno, segundo a utilização ou não da suplementação vitamino-mineral em mães e recém-nascidos.

\begin{tabular}{lccc}
\hline & $\begin{array}{c}\text { G1 } \\
\mathbf{( n : 3 4 )}\end{array}$ & $\begin{array}{c}\mathbf{G} \mathbf{2} \\
\mathbf{( n : 3 0 )}\end{array}$ & $\mathbf{p}$ \\
\hline Mãe & $0,45 \pm 0,27^{*}$ & $0,37 \pm 0,25^{*}$ & $0,18^{\star}$ \\
RN** & - & - & \\
\hline$*$ média \pm dp & $* *$ níveis indetectáveis & & \\
teste t de Student & & &
\end{tabular}

Tabela 6: Concentrações plasmáticas ( $\mathrm{mmol} / \mathrm{L}$ ) de $\beta \phi$ caroteno, segundo a utilização ou não da suplementação vitamino-mineral em mães e recém-nascidos.

\begin{tabular}{lccc}
\hline & $\begin{array}{c}\text { G1 } \\
(\mathbf{n}: 7)\end{array}$ & $\begin{array}{c}\text { G2 } \\
(\mathbf{n}: 7)\end{array}$ & $\mathbf{p}$ \\
\hline Mãe & $0,33 \pm 0,18^{*}$ & $0,39 \pm 0,30^{*}$ & $0,50^{*}$ \\
RN** & - & - & \\
\hline$*$ média $\pm d p$ & $* *$ níveis indetectáveis & teste t de Student &
\end{tabular}

O grupo de mães que receberam suplementação vitamino-mineral, tinha concentrações plasmáticas médias de $\alpha$ tocoferol (vitamina E) superiores aos do outro grupo. $\mathrm{Na}$ tabela 7 observa-se que embora não tenha ha- vido diferença entre as concentrações plasmáticas médias dos dois grupos de recémnascidos, os mesmos apresentavam concentrações plasmáticas médias de $\alpha$ tocoferol mais baixas do que os de suas mães.

Tabela 7: Concentrações plasmáticas (mmol / L) de $\alpha$ tocoferol, segundo a utilização ou não da suplementação vitamino-mineral em mães e recém-nascidos.

\begin{tabular}{lccc}
\hline & $\mathbf{G 1}$ & $\mathbf{G} \mathbf{2}$ & $\mathbf{p}$ \\
& $\mathbf{( n : 3 6 )}$ & $\mathbf{( n : 3 2 )}$ & \\
\hline Mãe & $11,7 \pm 4,92^{*}$ & $9,46 \pm 2,80^{*}$ & $\mathbf{0 , 0 2}{ }^{\bullet \bullet}$ \\
RNP & $2,06 \pm 0,81^{*}$ & $1,71 \pm 1,13^{*}$ & $\mathbf{0 , 9 8 *}$ \\
& $0,0001^{\star}$ & $0,0001^{\star}$ & \\
\hline
\end{tabular}

* Média \pm desvio padrão

- teste t de Student para amostras não independentes

- teste $\mathrm{t}$ de Student para amostras independentes

No que tange às concentrações plasmáticas de cobre (tabela 8), observou-se que as mães tinham concentrações plasmáticas médias significantemente maiores que as dos seus recém-nascidos, embora o comportamento em ambos os grupos, com e sem suplementação, não revelasse diferenças, tanto entre as mães quanto entre os recém-nascidos. 
Tabela 8: Concentrações plasmáticas médias $(\mathrm{mg} / \mathrm{mL})$ de cobre, segundo a utilização ou não da suplementação vitamino-mineral em mães e recém-nascidos.

\begin{tabular}{|c|c|c|c|}
\hline & $\begin{array}{c}\text { G1 } \\
(\mathrm{n}: 38)\end{array}$ & $\begin{array}{c}\mathrm{G} 2 \\
(\mathrm{n}: 35)\end{array}$ & p \\
\hline Mãe & $1,58 \pm 0,51^{*}$ & $1,48 \pm 0,46^{*}$ & $0,22^{\star \bullet}$ \\
\hline $\mathrm{RNp}$ & $\begin{array}{c}0,30 \pm 0,16^{*} \\
0,0001 \mathrm{~s}\end{array}$ & $\begin{array}{c}0,35 \pm 0,27 * \\
0,0001 \mathrm{~s}\end{array}$ & $0,92^{\star \star}$ \\
\hline
\end{tabular}

* Média \pm desvio padrão

- teste $\mathrm{t}$ de Stdent para amostras não independentes

* test $\mathrm{t}$ de Student para amostras independentes

Tabela 9: Correlação entre as concentrações plasmáticas dos micronutrientes das mães e recémnascidos nos dois grupos do estudo.

\begin{tabular}{lccc}
\hline & $\mathbf{n}^{*}$ & $\begin{array}{c}\text { Coeficiente de correlação de } \\
\text { Pearson(r) }\end{array}$ & $\mathbf{p}^{*}$ \\
\hline retinol (A) & 68 & 0,8 & 0,0001 \\
tocoferol & 68 & 0,4 & 0,0002 \\
cobre & 73 & 0,2 & 0,0381 \\
\hline
\end{tabular}

* pares mãe/RN

- Teste F (Fischer-Snedecor)

A análise da relação das concentrações plasmáticas médias dos micronutrientes das mães e dos recém-nascidos (tabela 9), independentemente do grupo a que pertenciam, revelou correlação significante estatisticamente para o retinol, $\alpha$ tocoferol e cobre, sendo que o maior coeficiente de Pearson foi observado para o retinol.

\section{DISCUSSÃO}

Antes de se efetuar comparações quanto aos nutrientes devem ser consideradas as características da casuística para verificar se existem diferenças entre os grupos, o que poderia dificultar as comparações efetuadas. A tabela 1 evidencia que não havia diferenças importantes entre os grupos, fazendo exceção apenas o tipo de parto, normal ou cesáreo, o que não interfere nos micronutrientes estudados.

Também quanto às características dos recém-nascidos não houve diferenças entre os dois grupos, o que indica que os mesmos eram comparáveis, independentemente das mães terem ou não recebido suplementação no decorrer da gestação.

No inquérito alimentar, em termos médios, as gestantes estudadas revelaram um padrão de ingestão calórica 50\% inferior às recomendações do "Food and Nutrition Board" de 1989, que considera como adequada uma ingestão média diária de 2200 calorias, acrescidas de 300 calorias/dia a partir do segundo trimestre de gestação. ${ }^{24}$ Não se evidenciaram diferenças significantes entre os dois grupos de estudo.

O retinol (vitamina A), a partir do inquérito alimentar verificou-se que as gestantes de ambos os grupos receberam quantida- 
des de retinol acima da recomendada. A análise da ingestão média diária em ambos os grupos não revelou diferenças estatisticamente significativas.

No que tange às concentrações plasmáticas, apesar de terem sido maiores nas mães que receberam suplementação, as médias encontradas eram baixas em ambos os grupos, quando comparadas aos valores de referência para mulheres não grávidas da mesma faixa de idade. Quanto aos recém-nascidos, as concentrações plasmáticas médias do retinol foram semelhantes entre os grupos, porém como o valor do $p$ observado foi muito próximo do nível de significância é possível que isto indique uma tendência a que os recém-nascidos também tenham algum benefício decorrente da suplementação que suas mães receberam, o que aqui não foi detectado, provavelmente em função do tamanho dos grupos de estudo. É importante salientar que a suplementação não foi suficiente para suprir os deficits de estoque e a maior necessidade de retinol decorrentes da gestacão. Já nas concentrações plasmáticas médias das mães em ambos os grupos, verificou-se que estas eram superiores às de seus recém-nascidos, fato semelhante ao descrito anteriormente na literatura ${ }^{7,11,15,25}$.

Já em relação à ingestão do licopeno e do $\beta$ caroteno, as necessidades nutricionais diárias ainda não estão definitivamente estabelecidas.

Neste estudo foram encontradas concentrações plasmáticas médias de 0,39 $\pm 0,30 \mathrm{umol} / \mathrm{L}$ em somente 7 mães de cada grupo, sendo que nas demais os níveis foram tão baixos que não permitiram sua dosagem. Nos grupos de recém-nascidos não foram detectados níveis séricos de $\beta$ caroteno. Este comportamento é sugestivo de que deve existir uma deficiência que, durante a gestação, pode vir a ter repercussões negativas para o concepto, particularmente se se fizer comparações com outro estudo que conseguiu detectar níveis séricos de $\beta$ caroteno em recém-nascidos, ainda que inferiores aos maternos ${ }^{7,12,25}$.

As concentrações plasmáticas médias maternas de licopeno não evidenciaram diferenças entre os dois grupos do estudo, sendo, inclusive, muito próximas e até superiores às referidas por vários autores ${ }^{7,12,25}$.

No que concerne aos recém-nascidos, apenas em um dos casos do G1 foi possível detectar concentrações plasmáticas séricas de licopeno. Deste modo o $\beta$ caroteno e o licopeno apresentaram comportamento semelhante ao do retinol, com concentrações plasmáticas nos recém-nascidos menores do que as maternas.

Quanto às concentrações plasmáticas de $\alpha$ tocoferol, o grupo de mães que recebeu suplementação apresentou média estatisticamente maior do que a do grupo de mães que não a receberam. Embora isto possa sugerir benefícios para as mães, com os níveis observados no presente estudo não havia influência nos recém-nascidos, o que é compatível com o citado por outros auores ${ }^{7,26}$.

As concentrações plasmáticas médias de cobre nos grupos de mães foram semelhantes; com ou sem suplementação. O mesmo ocorrendo com os grupos de recém-nascidos, sugerindo que a sua suplementação, nos moldes que foi realizada, não foi suficiente para influenciar os níveis séricos tanto das mães como dos recém-nascidos.

As mães de ambos os grupos apresentaram concentrações plasmáticas médias de cobre maiores do que as dos recém-nascidos. Mesmo assim as concentrações plasmáticas médias de cobre observadas tanto para os grupos de mães quanto para os recém-nascidos, foram menores do que a média aceita como normal, sugerindo uma deficiência que, mesmo com a suplementação, não chegou a ser evitada ${ }^{20,26,27}$.

No seu conjunto, os baixos níveis de micronutrientes detectados concordam com as dificuldades existentes para se estudar as correlações entre os níveis plasmáticos maternos e os dos recém-nascidos. Apesar disto, obser- 
vou-se uma correlação estatisticamente significante mãe/RN para retinol, $\alpha$ tocoferol e cobre, mas apenas o retinol evidenciou uma elevada correlação entre ambos.

Existe uma provável deficiência de retinol, $\beta$ caroteno, licopeno, $\alpha$ tocoferol e cobre em gestantes, mesmo entre as que recebem suplementação, com níveis ainda mais baixos nos recém-nascidos. Não há aparentemente consequências clínicas imediatas disto, pelo menos no momento do parto, não sendo impossível que, entretanto, venham a ter expressão clínica no futuro.

As concentrações nos recém-nascidos apresentam a mesma tendência das mães, com correlação, mesmo que baixa, com os níveis maternos para a maioria dos micronutrientes aqui estudados.

\section{REFERÊNCIAS}

1. Mejia LA, Arroyave G. Las Vitaminas. In: Carraza FR, Marcondes E. Nutrição Clínica em Pediatria. 1991.São Paulo, Sarvier.

2. Bates CJ, Prentice A. Breast milk as a souce of vitamins, essential minerals and trace elements. Pharmac. Ther.1994. v 62, pp.193-220.

3. Knight EM, Spurlock DG, Edwards $\mathrm{CH}$, Johnson AA, Oyemade UJ, Cole OJ, West WL, Manning M, James H, Laryea H. Biochemical profile of African American women during three trimesters of pregnancy and at delivery. J. Nutr. Supplement 1994.v.24, p.943-53.

4. Poppit SD, Prentice AM. Maternal nutrition deficiencies in develolping countries. In: Baum J.D. Birth risks.1993. New York. Raven Press, P.71-82.

5. Prentice AM, Goldberg GR. Energy adaptations in human pregnancy: limits and long-term consequences. Am. J. Clin.
É plausível considerar que as diferenças observadas entre as concentrações maternas e dos recém-nascidos sejam um aspecto decorrente da fisiologia da relação mãe/ feto. Entretanto, é possível pressupor a ausência de um mecanismo de proteção do concepto frente às possíveis deficiências de retinol, $\beta$ caroteno, licopeno, $\alpha$ tocoferol e cobre, apresentadas pelas mães no decorrer da gravidez.

A pequena influência exercida pela suplementação sobre as concentrações plamá ticas das mães aliada ao fato da baixa ingestão destes micronutrientes que as mesmas apresentam, indica a premente necessidade de se realizar ulteriores estudos quanto à educação alimentar e também quanto à forma de suplementação que deveria ser realizada durante a gestação, objetivando prevenir deficiências neste período da vida.

Nutr. Supplement 2000. v.71, p.1226-32. Supplement 2000.

6. Czeizel AE, Rockenbauer M. Prevention of congenital abnormalities by vitamin A. Internat. J. Vit. Nutr. Res. 1998.v.68, p.219-31.

7. Sapin V, Alexandre MC, Chaïb $\mathrm{S}$ Bournazeau JA, Sauvant P, Borel P, Jacquetin B, Grolier P, Lémery D, Dastugue B,Azaïs-braesco V. Effect of vitamin A status at the end of term pregnancy on the saturation of retinol binding protein with retinol. Am. J. Clin. Nutr. 2000. v.71, p.537-43.

8. Rothman K.J, Moore L.L, Singer M.R, Nguyen ODT, Mannino S, Milunsky A. Teratogenicity of high vitamin A intake. N. Engl. J. Med. 1995. v.333, p.369-73.

9. Mastroiacovo P, Mazzone T, Addis A, Elephant E, Carlier P, Vial T, Garbis H, Robert E, Bonati M, Ornoy A, Finardi A, Schaffer C, Caramelli L, Rodríguezpinilla, E, Clement M. High Vitamin A intake in early pregnancy and major 
malformations: a multicenter prospective controlled study. Teratology. 1999. v.59, p.7-11.

10. Antipatis C, Grant G, Ashworth CJ. Moderate maternal vitamin A deficiency affects perinatal organ growth and development in rats. Br. J. Nutr. 2000 84, p.125-32.

11. Ghebremeskel K, Burns L, Burden TJ, Harbige L, Costeloe K, Powell JJ, Crawford M. Vitamin A and related essential nutrients in cord blood: relationships with anthropometric measurements at birth. Early Hum. Dev. 1994. v.39, p.177-88.

12. Daudu P.A, Kelley DS, Taylor PC, Burri $\mathrm{BJ}, \mathrm{Wu}$ MM. Effect of a low b-carotene diet on the immune functions of adult women. Am. J. Clin. Nutr. 1994.v.60, p.969-72.

13. Gester H. The potencial role of lycopene for human health. J. Am. Coll. Nutr. 1997. v.16, p.109-126.

14. Stahls W, Sies H. Uptake of licopene and its geometrical isomers is greater from heat-processed than from unprocessed tomato juice in in humans. J. Nutr.1992. v. 122, p 2161-6.

15. Azaïs-Braesco V, Pascal G. Vitamin A in pregnancy: requirements and safety limits. Am. J. Clin. Nutr. Supplement 2000. v. 71, p.1325-33.

16. Abbassi S, Ludomirski A, Bhutani VK, Weiner S, Johnson L. Maternal and fetal plasma vitamin $\mathrm{E}$ to total lipid ratio and fetal RBC antioxidant function during gestational development. J Am Coll Nutr. 1990.v.9, p 314-9.

17. Jain SK, Wise R, Bocchini JJ. Vitamin E and vitamin E-quinone levels in red blood cells and plasma of newborn infants and their mothers. J. Am. Coll. Nutr. 1996.v.15, p.44-8.
18. Akyol D, Mungan T, Görkemli H, Nuhoglu G. Maternal levels of vitamin E in normal and preeclamptic pregnancy. Arch. Gynecol. Obstet. 2000. v.263, p.151-5.

19. Tong KK, Hannigan BM, Mckerr, G. The effects of copper deficiency on women lymphoid and myeloid cells: an in vitro model. Br. J. Nutr. 1996. v.75, p.97-108.

20. Bellido MC, Vázquez AMM, Ferriz MB, Román MC, Cos SR, Barrios JMT. Cobre en el período neonatal. Relaciones materno-fetales. An. Esp. Pediatr. 1996.v.44, p.145-8.

21. Henkin RI, Marshall JR, Meret S. Maternal-fetal metabolism of copper and zinc at term. Am.J.Obstet. Gynecol. 1971.v.110, p 131-4.

22. Halmesmaki E, Alfthan G,Ylikorkala O. Selenium in pregnancy: effect of maternal drinking. Obstet. Gynecol. 1986.v.68, p 602-5.

23. Noubah AM, Al-awqati MA. Ultrafiltrable copper and related analytes in maternal and cord bloob. Cin. Chem. 1990.v.36, p 8604.

24. Recommended dietary allowances. National Research Council. Washington (DC): National Academy Press 1989.

25. Yeum K, Ferland G, Patry J, Russell RM. Relationship of Plasma Carotenoids, Retinol and Tocopherols in Mothers and Newborn Infants. J. Am. Coll. Nutr. 1998. v.17, p.442-7.

26. Arnaud J, Preziosi P, Mashako, L, Galan P, Nsibu C, Favier A,Kapongo C, Hercberg $S$. Serum trace elements in Zairian mothers and their newborns. Eur. J. Clin. Nutr. 1994. v.48, p.341-8.

27. Lee SH, Lancey R, Montaser A, Madani $\mathrm{N}$, Linder MC. Ceruloplasmin and copper transport during the latter part of gestation in the rat. Proc. Soc. Exp. Biol. Med. 1993.v.203, p.428-39. 
28. Scaife AR, McNeill G, Campbell DM, Martindale S, Devereux G, Seaton A. Maternal intake of antioxidant vitamins in pregnancy in relation to maternal and plasma levels at delivery. British Journal of Nutrtion 2006.v.95, p 771-778.

29. S. Shah PS, Arne Ohlsson A. On behalf of the Knowledge Synthesis Group on Determinants of Low Birth Weight and Preterm Births. Effects of prenatal multimicronutrients supplementation on pregnancy outcomes: a metaanalyses CMAJ 2009.180(12):E99-E108

30. Christian P, Khatry SK, Katz J, Pradhan EK, LeClerq SC, Shrestha SR, Adhikari RK, Sommer A, West Jr KP. Effects of alternative maternal micronutrient supplements on low birth weight in rural Nepal: double blind randomised community trial. BMJ. 2003. v. 326, p 2-6.

Recebido em 12 de setembro de 2009 Modificado em 14 de novembro de 2009 Aceito em 30 de janeiro de 2010 